\title{
Targeting CD38-Expressing Multiple Myeloma and Burkitt Lymphoma Cells In Vitro with Nanobody-Based Chimeric Antigen Receptors (Nb-CARs)
}

\author{
Julia Hambach ${ }^{1,2}$, Kristoffer Riecken ${ }^{3,4} \oplus$, Sophia Cichutek ${ }^{3,4}$, Kerstin Schütze ${ }^{1,2}$, \\ Birte Albrecht 1,2, Katharina Petry ${ }^{1,2}$, Jana Larissa Röckendorf ${ }^{1,2}$, Natalie Baum ${ }^{1,2}$, \\ Nicolaus Kröger ${ }^{4}$, Timon Hansen ${ }^{5}$, Gunter Schuch ${ }^{5}$, Friedrich Haag ${ }^{1}$, Gerhard Adam ${ }^{2}$, \\ Boris Fehse ${ }^{3,4, *(D)}$, Peter Bannas ${ }^{2, *(D)}$ and Friedrich Koch-Nolte ${ }^{1, *}$ \\ 1 Institute of Immunology, University Medical Center Hamburg-Eppendorf (UKE), 20246 Hamburg, Germany; \\ j.hambach@uke.de (J.H.); ke.schuetze@uke.de (K.S.); bi.albrecht@uke.de (B.A.); k.petry@uke.de (K.P.); \\ Jana.Roeckendorf@stud.uke.uni-hamburg.de (J.L.R.); n.baum@uke.de (N.B.); haag@uke.de (F.H.) \\ 2 Department of Diagnostic and Interventional Radiology and Nuclear Medicine, UKE, 20246 Hamburg, \\ Germany; g.adam@uke.de \\ 3 Research Department Cell and Gene Therapy, UKE, 20246 Hamburg, Germany; \\ k.riecken@uke.uni-hamburg.de (K.R.); sophiac@gmx.net (S.C.) \\ 4 Department of Stem Cell Transplantation, UKE, 20246 Hamburg, Germany; n.kroeger@uke.de \\ 5 Hematological-Oncology Center Altona, 22767 Hamburg, Germany; \\ Timon.Hansen@hopa-hamburg.de (T.H.); gunter.schuch@hopa-hamburg.de (G.S.) \\ * Correspondence: fehse@uke.de (B.F.); p.bannas@uke.de (P.B.); nolte@uke.de (F.K.-N.)
}

Received: 28 November 2019; Accepted: 22 January 2020; Published: 29 January 2020

check for updates

\begin{abstract}
The NAD-hydrolyzing ecto-enzyme CD38 is overexpressed by multiple myeloma and other hematological malignancies. We recently generated CD38-specific nanobodies, single immunoglobulin variable domains derived from heavy-chain antibodies naturally occurring in llamas. Nanobodies exhibit high solubility and stability, allowing easy reformatting into recombinant fusion proteins. Here we explore the utility of CD38-specific nanobodies as ligands for nanobody-based chimeric antigen receptors ( $\mathrm{Nb}$-CARs). We cloned retroviral expression vectors for CD38-specific $\mathrm{Nb}$-CARs. The human natural killer cell line NK-92 was transduced to stably express these $\mathrm{Nb}$-CARs. As target cells we used CD38-expressing as well as CRISPR/Cas9-generated CD38-deficient tumor cell lines (CA-46, LP-1, and Daudi) transduced with firefly luciferase. With these effector and target cells we established luminescence and flow-cytometry CAR-dependent cellular cytotoxicity assays (CARDCCs). Finally, the cytotoxic efficacy of Nb-CAR NK-92 cells was tested on primary patient-derived CD38-expressing multiple myeloma cells. NK-92 cells expressing CD38-specific $\mathrm{Nb}$-CARs specifically lysed CD38-expressing but not CD38-deficient tumor cell lines. Moreover, the $\mathrm{Nb}$-CAR-NK cells effectively depleted CD38-expressing multiple myeloma cells in primary human bone marrow samples. Our results demonstrate efficacy of $\mathrm{Nb}$-CARs in vitro. The potential clinical efficacy of $\mathrm{Nb}$-CARs in vivo remains to be evaluated.
\end{abstract}

Keywords: CD38; nanobody; chimeric antigen receptor; NK-92 cells; cellular cytotoxicity assays; luciferase; heavy chain antibody; multiple myeloma 


\section{Introduction}

The NAD-hydrolyzing ecto-enzyme CD38 is overexpressed by multiple myeloma and other hematological malignancies [1-3]. CD38 has emerged as a promising target for therapy with cytotoxic antibodies. Three monoclonal antibodies have recently entered the clinic (daratumumab, isatuximab, and MOR202) for treatment of multiple myeloma (Figure 1a) [4-6].

(a)

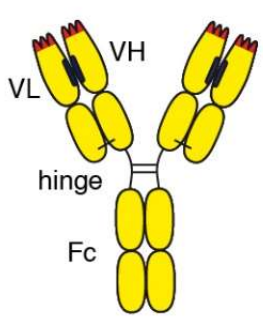

conventional $\mathrm{Ab}$

(Ab)

(b)

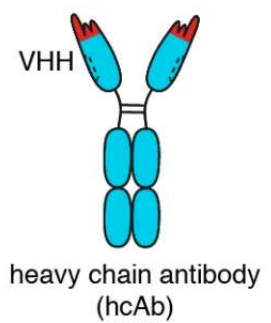

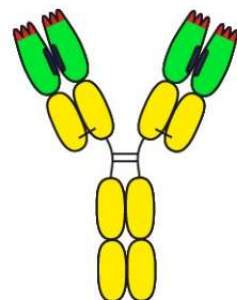

chimeric $\mathrm{Ab}$

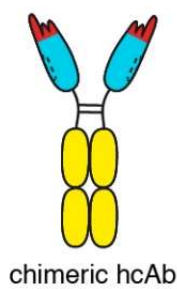

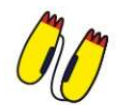

single-chain variable Fragment (scFv)

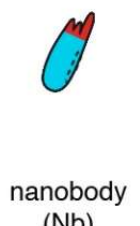

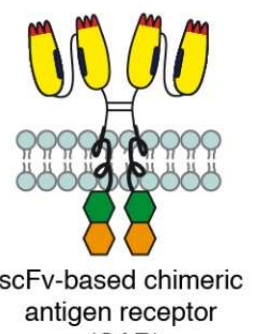
(CAR)

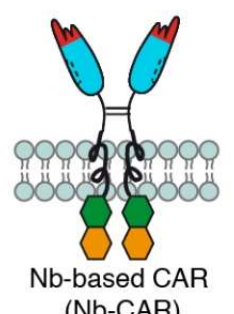

Figure 1. Comparison of conventional and nanobody-based chimeric antigen receptors. (a) Schematic diagrams of a conventional antibody $(\mathrm{Ab})$, a chimeric antibody, a single chain variable fragment (scFv), and conventional scFv-based chimeric antigen receptor (CAR). Examples of conventional and chimeric CD38-specific Abs include daratumumab and MOR202 (conventional Abs) and isatuximab (chimeric $\mathrm{Ab}$ ). (b) Schematic diagrams of a camelid heavy chain antibody (hcAb), a chimeric hcAb, a nanobody $(\mathrm{Nb})$ and a $\mathrm{Nb}$-based chimeric antigen receptor ( $\mathrm{Nb}-\mathrm{CAR})$.

The variable domains of conventional antibodies (VH and $\mathrm{VL}$ ) interact via hydrophobic patches (indicated in black) that help to correctly align the two domains for target binding. The hydrophobic nature of this interaction and its relatively low affinity, however, render scFvs unstable and sticky (Figure 1a). Nanobodies are single variable immunoglobulin domains derived from heavy chain antibodies (hcAbs) that naturally occur in llamas, dromedaries, and other camelids (Figure 1b) $[7,8]$. The mutation(s) that led to deletion of the $\mathrm{CH} 1$ domain in heavy chain antibodies must have occurred $>50$ million years ago, i.e., in a common ancestor of today's extant camelids (dromedary, camel, alpaca, llama) [9]. The cluster of $\mathrm{V}$ genes in the IgH locus of extant camelids encodes both, highly soluble $(\mathrm{VHH})$ and less soluble $(\mathrm{VH})$ domains. The former occur in the vast majority of camelid hcAbs, the latter in camelid conventional Abs akin to those of mice and men [9]. Camelid VHH domains apparently have been shaped by evolution for high solubility and stability as a single domain. This renders nanobodies particularly suited for reformatting in a LEGO-block like fashion into fusion proteins, including dimers or multimers of nanobodies, chimeric llama/human $\mathrm{Nb}$-hcAbs, biparatopic hcAbs, and Nb-based CARs (Nb-CARs) $[10,11]$. Dimerization and multimerization of nanobodies has been used to increase the binding strength and the specificity of the constructs $[10,11]$.

From immunized llamas, we previously isolated a panel of CD38-specific nanobodies [12,13]. We have shown that these nanobodies can be used to construct $\mathrm{Nb}$-hcAbs, some of which exhibit more potent antibody-dependent cellular cytotoxicity (ADCC) than daratumumab (Figure 2) $[10,14,15]$. 


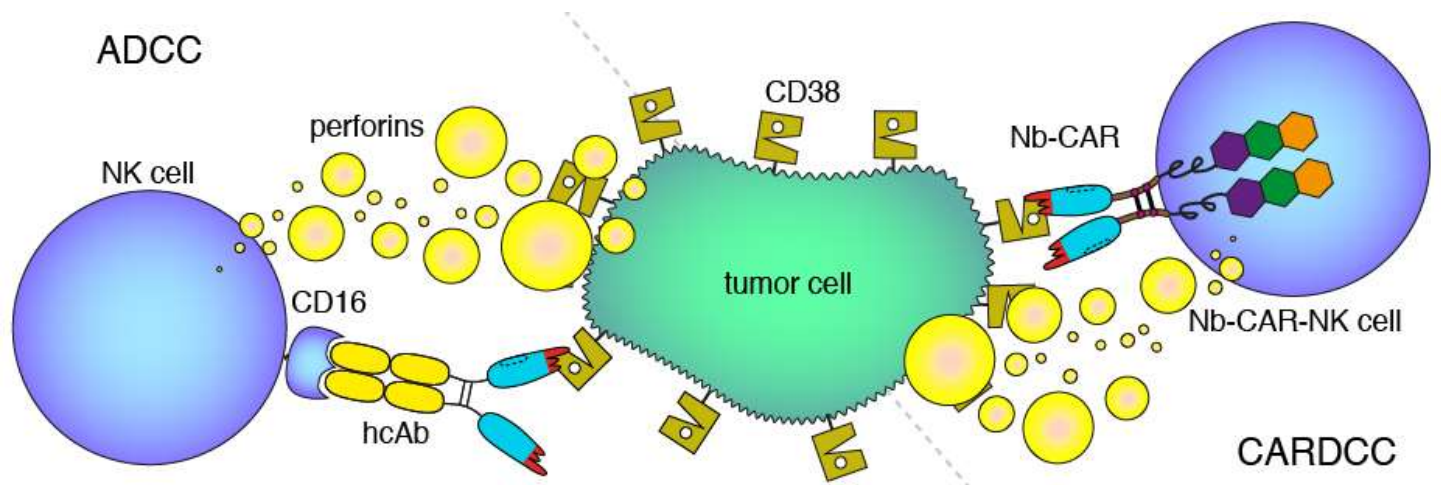

Figure 2. Antibody-and CAR-dependent cellular cytotoxicity. Schematic diagrams of antibody-dependent cellular cytotoxicity (ADCC, left) and chimeric antigen-receptor dependent cellular cytotoxicity (CARDCC, right) mediated by CD38-specific hcAbs and Nb-CARs, respectively. ADCC and CARDCC are mediated by perforins, pore-forming cytolytic proteins released from activated NK cells. Perforins are depicted here schematically as yellow bubbles.

The aim of the current study was to explore the utility of CD38-specific nanobodies as ligands for Nb-CARs. To this end, we stably transduced the human natural killer cell line NK-92 [16] with CD38-specific and control Nb-CARs. We chose NK-92 cells as effector cells rather than T cells because of their potential use as an "off-the-shelf" reagent. As target cells, we chose established multiple myeloma (LP-1, RPMI-8226) and Burkitt lymphoma cell lines (CA-46, Daudi) with known high cell surface levels of CD38. With these cells, we established CAR-dependent cellular cytotoxicity assays (CARDCC) (Figure 2), using luminescence and flow-cytometry to monitor the killing of target cells (see below). Our results indicate that $\mathrm{Nb}$-CARs, indeed, may provide a basis for clinical development of novel therapeutics to target CD38-expressing tumor cells.

\section{Materials and Methods}

\subsection{Cell Lines}

The following human cell lines were obtained from the German Collection of Microorganisms and Cell Culture (DSMZ, Braunschweig, Germany): NK-92 (natural killer cell line), Daudi and CA-46 (Burkitt lymphoma cell lines), LP-1 and RPMI-8226 (myeloma cell lines). Cell lines (CA-46 luc, Daudi luc, LP-1 luc) stably expressing the luc2 variant of Photinus pyralis luciferase (Promega, Madison, WI, USA) under control of the spleen-focus-forming virus U3 region (SFFV promoter) were generated by lentiviral transduction as described $[17,18]$. Transduced cells were selected in culture medium containing $1 \mu \mathrm{g} / \mathrm{mL}$ puromycin and subsequently sorted by FACS based on eGFP expression (on a FACS Aria III, BD Biosciences, Heidelberg, Germany). Sorted cells were kept in culture and luciferase-expression was controlled regularly following addition of luciferin using a luminometric plate reader.

As specificity controls, we generated CD38-deficient variants of LP-1 luc, CA-46 luc, Daudi luc, and NK-92. In order to prevent expression of CD38, rather than to merely reduce its expression, we used CRISPR/Cas9 technology (sc-401117-NIC, Santa Cruz Biotechnology, Dallas, TX, USA) rather than shRNA technology. Cells were stained for CD38 using the AlexaFluor647-conjugated JK36 nanobody $\left(\mathrm{JK} 36^{\mathrm{AF} 647}\right.$ ) and cells were FACS-sorted for lack of cell surface CD38. The sorted cells were maintained in culture and CD38 cell surface levels were controlled regularly using JK36 ${ }^{\mathrm{AF} 647}$. 


\subsection{Generation of $\mathrm{Nb}$-CARs}

The human CD38-specific nanobodies WF211, MU1067, JK36 and the ARTC2.2-specific control nanobody s-14 were generated from immunized llamas as described previously $[13,19]$. In the text, we use the full designation for these nanobodies. For the sake of clarity, we use abbreviated designations for $\mathrm{Nb}$-CARs, e.g., Nb1067-CAR and Nb14-CAR instead of NbMU1067-CAR and Nbs-14-CAR. The nanobody coding region was fused by gene synthesis downstream of the signal sequence of $\mathrm{VH}$ and upstream of a strep-tag, the hinge region of human IgG4, the transmembrane and membrane-proximal ITAM domains of human CD28, the ITAM domain of human 4-1BB, and the C-terminal signaling domain of CD3 $\zeta$. This cassette was cloned into the gamma-retroviral vector pRSF91.iB.pre* (a derivative of pRSF91.GFP.pre* [20]) upstream of an EMCV internal ribosomal entry site (IRES) and the coding region for blue-fluorescent protein (mTagBFP). HEK293 cells were transiently transfected with the combination of the pRSF91.Nb-CAR.iB.pre* coding for the CAR and packaging plasmids pcDNA3.MLVgp [21] and phCMV-GALV ${ }_{C 4070 A}$-env to obtain cell-free supernatants containing retroviral particles encoding $\mathrm{Nb}$-CARs. Vector titers were then determined on HEK293T cells as described previously [18].

\subsection{Stable Transduction of NK-92 Cells with Nb-CARs}

NK-92 cells stably expressing Nb-CARs were generated by retroviral transduction. Transduction was carried out in a 24-well plate with 50,000 cells in $500 \mu \mathrm{L}$ medium per well by addition of $300 \mu \mathrm{L}$ viral particle-containing supernatant in the presence of $8 \mu \mathrm{g} / \mathrm{mL}$ hexadimethrine bromide and subsequent spin-inoculation for $1 \mathrm{~h}$ at $1000 \times g$ and $25^{\circ} \mathrm{C}$. Stably transduced cells were FACS-sorted based on mTagBFP-expression. CAR-expression by these cells was controlled regularly by staining of cells with AlexaFluor647-conjugated recombinant ectodomains of CD38 and ARTC2.2. The initial transduction efficiency was below $30 \%$; cell sorting resulted in stable expression of the $\mathrm{Nb}-\mathrm{CAR}$ by more than $95 \%$ of cells. The fluorochrome-conjugated ecto-domains of CD38 and ARTC2.2 served as both, positive and negative quality controls for determining the cell surface levels of target-specific $\mathrm{Nb}-\mathrm{CARs}$.

\subsection{Production of Alexa Fluor 647-Labeled CD38 and ARTC2.2}

The myc-his-tagged extracellular domains of CD38 (aa46-300) and ARTC2.2 (aa20-261) were produced in transiently transfected HEK-6E cells cultivated in serum-free medium. Six days post transfection supernatants were harvested and cleared by centrifugation. The myc-his-tagged proteins were purified by immobilized metal affinity chromatography using Ni-NTA agarose (Sigma, St Louis, MO, USA). Fluorochrome-labelling was performed using NHS esters according to the manufacturer's instructions (Alexa Fluor 647 Succinimidyl Ester, Invitrogen, Karlsbad, CA, USA).

\subsection{Luminescence CARDCC Assays}

CA-46 luc, Daudi luc, and LP-1 luc cells were co-incubated with NK-92-CAR for $4 \mathrm{~h}$ at $37^{\circ} \mathrm{C}$ at the indicated ratios in $\alpha$ MEM culture medium supplemented with $10 \%$ fetal calf serum (FCS), $10 \%$ horse serum, $5 \mathrm{mM}$ glutamine, and $5 \mathrm{ng} / \mathrm{mL}$ interleukin 2 (IL-2 Proleukin-S, Novartis, Basel, Switzerland). D-luciferin (Biosynth, Staad, Switzerland) was added as substrate $(75 \mu \mathrm{g} / \mathrm{mL}$ ) for $20 \mathrm{~min}$ and bioluminescence-intensity (BLI) was measured with a microplate reader (Victor ${ }^{3}$, Perkin Elmer, Boston, MA, USA). 


\subsection{Flow Cytometric CARDCC Assays}

Target cells were fluorescently pre-labeled by incubation with AlexaFluor647, effector cells by incubation with eFluor450. Cells were washed and co-incubated at the indicated E:T-ratios at $37^{\circ} \mathrm{C}$ for the indicated time-periods. Dead cells were stained with propidium iodide (PI, Invitrogen, WA, USA) or Pacific Orange succinimidyl ester (PacO, Thermo-Fisher Scientific, Waltham, MA, USA) before analysis of cells by flow cytometry (BD FACS Celesta/Becton Dickinson). Percentage of cells was calculated as follows: $\%$ lysis [\%] = $1-$ (cells [sample]/ cells [sample with control CAR] $) \times 100 \%$.

\subsection{CARDCC Assays with Primary Human Bone Marrow Samples}

Fresh bone marrow aspirates were obtained from patients after Institutional Review-Board-approved consent (PV5505). Bone marrow mononuclear cells (BM-MNCs) were prepared by Ficoll-Paque density gradient centrifugation of bone marrow aspirates and subsequent depletion of remaining erythrocytes using red blood cell lysis buffer $(\mathrm{NH} 4 \mathrm{Cl}+\mathrm{KHCO} 3+$ EDTA). BM-MNCs were co-incubated with eFluor450-labeled NK-92 Nb-CAR cells at an effector to target ratio [E:T] of 1:1 for $4 \mathrm{~h}$ at $37^{\circ} \mathrm{C}$ in $\alpha$ MEM culture medium (see above). Cells were then stained with a panel of fluorochrome-conjugated antibodies (CD38, CD45, CD138/229, CD269/CD319/CD56, CD19) and PacO and analyzed via flow cytometry. We did not use CD138 in these four hour assays because of the known instability of this marker on the cell surface of MM cells [22]. Staining of CD38 was achieved with Alexa Fluor 647-conjugated nanobodies that bind independently of the nanobody contained in the CAR: JK36 ${ }^{\mathrm{AF} 647}$ or MU523 ${ }^{\mathrm{AF} 647}$ for Nb211-CAR, MU523 ${ }^{\mathrm{AF} 647}$ or WF211 ${ }^{\mathrm{AF} 647}$ for Nb36-CAR, and JK36 ${ }^{\mathrm{AF} 647}$ or WF211 ${ }^{\mathrm{AF} 647}$ for Nb1067-CAR. An FSC threshold was set to exclude debris while including the population of small CD19 ${ }^{+}$B cells. NK-92 cells and dead cells were excluded via staining by eFluor450 and Pacific Orange, respectively. MM cells were identified by high co-expression of CD38 and CD56 or CD319. Numbers of MM cells were determined using CountBright absolute counting beads (Invitrogen, Karlsbad, CA, USA). Percentage of surviving MM cells was calculated as follows: Percent of survival [\%] $=(\mathrm{MM}$ cell number per $\mu \mathrm{L}$ [NK-92-CAR-treated sample]/MM cell number per $\mu \mathrm{L}$ [untreated sample] $\times 100 \%$. Significance between CD38-specific Nb-CAR-NK and the control Nb-CAR-NK was calculated using unpaired T-test (GraphPad Prism, GraphPad Software, CA, USA).

\section{Results}

\subsection{Generation of CD38-Deficient Cell Lines and Lentiviral Transduction of CD38 ${ }^{+}$and $C D 38^{-}$Cell Lines with Luciferase}

CD38 is overexpressed by several established human tumor cell lines, including LP-1 multiple myeloma, CA-46 and Daudi Burkitt lymphoma, and NK-92 natural killer cell lymphoma [1-3]. As specificity controls, we inactivated the CD38 gene in these cell lines using CRISPR/Cas9 technology. Cells were monitored for cell surface expression of CD38 using Alexa Fluor 647-conjugated CD38-specific nanobodies and CD38-deficient cells were FACS sorted for lack of CD38 cell surface expression (Figure 3a). The results confirm high expression of CD38 by parental cells and lack of CD38 expression by CD38ko cells. Similar results were obtained with Daudi luc cells (not shown). 
(a)

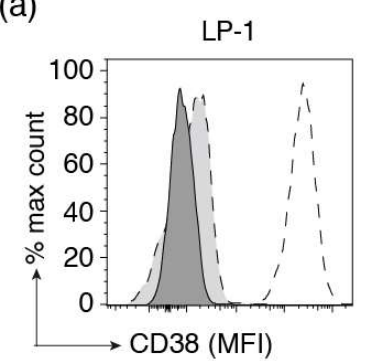

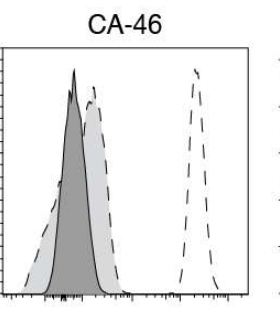

$\square$ unstained $\left.\Sigma_{-}^{-}\right]$luc

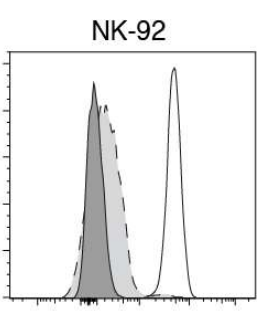

CD38ko $\square$ parental (b)

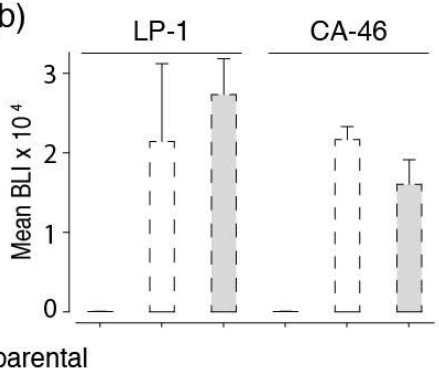

Figure 3. Inactivation of CD38 and stable expression of luciferase in established cell lines. (a) The CD38 gene was inactivated in the indicated tumor cell lines using CRISPR/Cas9 technology to provide specificity controls. Cells were FACS-sorted for lack of CD38 cell surface expression. Parental and CD38ko cells were incubated for $30 \mathrm{~min}$ at $4{ }^{\circ} \mathrm{C}$ with Alexa Fluor 647-conjugated $\mathrm{Nb}$ JK36 before analysis by flow cytometry. Results are representative for three similar experiments. (b) LP-1 and CA-46 cells and their corresponding CD38ko cell lines were lentivirally transduced with luc-GFP, encoding luciferase and GFP separated by an internal ribosomal entry site. GFP was used for cell sorting, luciferase was used for monitoring cell vitality by bio-luminometry. Cells were FACS-sorted for high expression of GFP. Triplicate samples of parental luc-GFP-expressing cells were incubated in a 96 well plate for $20 \mathrm{~min}$ at $24{ }^{\circ} \mathrm{C}$ with luciferin before analysis for luminescence with a plate reader. Results are representative for two similar experiments.

To permit luminescence-based cellular cytotoxicity assays, we stably transduced LP-1, CA-46, and Daudi cells with firefly luciferase and GFP. Cells were FACS sorted for high levels of GFP expression. Cells were monitored for luciferase activity following addition of luciferin using a luminescence plate reader. The results show lack of detectable bioluminescence by parental cells and very high levels of BLI signals in all luciferase-transduced cell lines (CD38 ${ }^{+}$and CD38ko cells) (Figure 3b). Killing assays revealed a high dynamic range of BLI signals in all luciferase-transduced cell lines (see below). Similar results were obtained with Daudi luc cells (not shown).

\subsection{Generation of Nanobody-Based Chimeric Antigen Receptors (Nb-CARs) and Transduction of NK-92 Cells with $\mathrm{Nb}$-CARs}

Nanobodies WF211, MU1067, and JK36 bind to three distinct, non-overlapping epitopes on CD38, designated E1, E2, and E3, respectively [13]. MU1067 inhibits the enzymatic activity of CD38, while WF211 and JK36 do not inhibit or enhance its activity [13]. The respective epitopes can be inferred from published crystal structures of CD38 in complex with nanobodies MU375 and MU551 and the Fab fragment of isatuximab (Figure 4a). WF211 competes for binding to CD38 with MU551 (E1), MU1067 competes for binding with MU375 (E2). JK36 binds to a third, non-overlapping epitope (designated E3) and competes neither with WF211 nor with MU1067 for binding to CD38. The Fab fragment of isatuximab also binds to an epitope that does not overlap with epitopes E1 and E2 (Figure 4a). It is possible, but not yet established experimentally that JK36 binds to a similar epitope as isatuximab.

For construction of $\mathrm{Nb}$-CARs, we fused each of the three CD38-specific nanobodies and a control nanobody (the ARTC2.2-specific nanobody s-14) genetically to the components of a classic third-generation CAR, i.e., the hinge region of IgG4, the transmembrane and intracellular ITAM domains of CD28, and the cytosolic signaling domains of 4-1BB and CD3 $\zeta$ (Figure 4b). We cloned these $\mathrm{Nb}$-CAR encoding cassettes into a retroviral vector upstream of an IRES followed by the coding region for blue fluorescent protein (BFP). NK-92-CD38ko cells were stably transduced with these vectors and cells were sorted for high levels BFP expression and for high levels of cell surface $\mathrm{Nb}$-CAR.

In order to detect cell surface levels of $\mathrm{Nb}$-CARs, we monitored cell surface levels of the respective $\mathrm{Nb}$-CARs by flow cytometry using the soluble ecto-domains of CD38 and ARTC2.2 conjugated to AlexaFluor647 (Figure 4c). The results show that CD38 ${ }^{647}$ specifically binds to NK-92 cells transduced with CD38-specific $\mathrm{Nb}$-CARs but not to cells transduced with the ARTC2.2-specific $\mathrm{Nb}$-CAR, whereas 
ARTC2.2 ${ }^{647}$ binds to NK-92 cells transduced with the ARTC2.2-specific Nb-CAR but not to NK-92 cells transduced with any of the three CD38-specific $\mathrm{Nb}$-CARs.

(a)

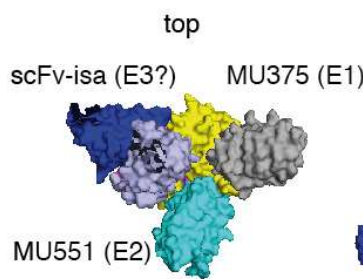

bottom side

(b)

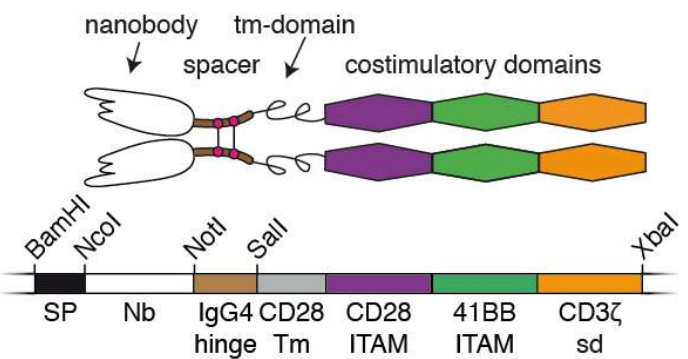

(c)
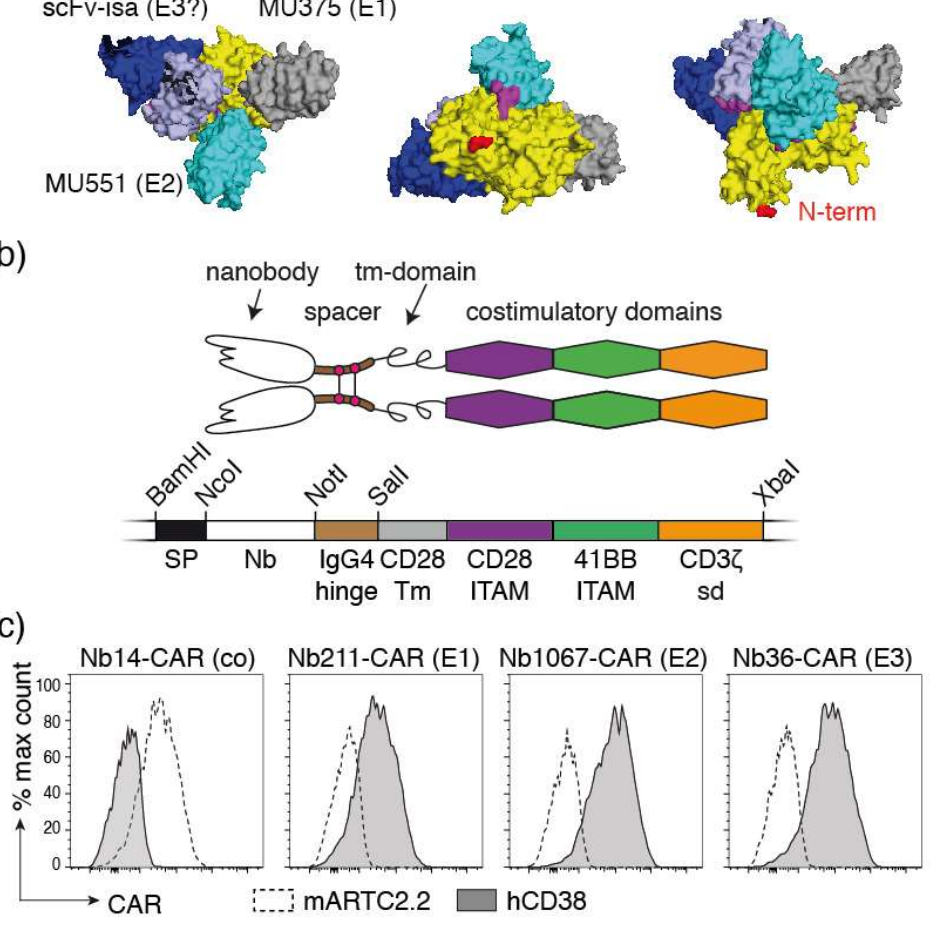

Figure 4. NK-92 cells stably expressing Nb-CARs. (a) 3D model of the extracellular domain of CD38 (yellow) in complex with two nanobodies (MU375 grey, and MU551 cyan) and the scFv of isatuximab (VH and VL in dark and light blue, respectively). The N-terminal amino acid of CD38 (depicted in red) connects to the membrane proximal amino acids and transmembrane domain. The model is presented from three points of view: the putative membrane-proximal side is designated "bottom," the membrane distal side "top" and the putative view parallel to the plane of the cell membrane "side." WF211 binds to the same epitope as MU375 (designated E1), MU1067 to the same epitope as MU551 (E2), and JK36 to a third epitope (E3). It is likely, but not yet confirmed experimentally, that JK36 and isatuximab bind to a similar epitope. (b) Schematic diagram of a Nb-CAR. The coding region for the $\mathrm{Nb}-\mathrm{CAR}$ was assembled by gene synthesis. The indicated restriction enzyme recognition sites were incorporated to allow flexible exchange of the corresponding elements. SP: signal peptide; $\mathrm{Nb}$ : nanobody, tm: transmembrane; ITAM immunoreceptor tyrosine-based activation motif, sd: signaling domain. (c) NK-92 cells were stably transduced with the indicated Nb-CARs and sorted for high expression of blue fluorescent protein BFP, which was encoded downstream of an IRES sequence behind the coding region for the $\mathrm{Nb}$-CAR. Cells were incubated with the fluorochrome-conjugated recombinant extracellular domains of mouse ARTC2.2 or human CD38 before analysis by flow cytometry. Results are representative for three similar experiments. 


\subsection{Luminescence-Based CAR-Dependent Cellular Cytotoxicity Assay (CARDCC)}

In order to test whether $\mathrm{Nb}-\mathrm{CAR}-\mathrm{NK}$ cells specifically lyse CD38-expressing tumor cells, we co-incubated $\mathrm{Nb}$-CAR-NK cells with luciferase-transduced CA- 46 cells for $4 \mathrm{~h}$ at $37^{\circ} \mathrm{C}$ before addition of luciferin. Luciferase activity was then monitored with a luminescence plate reader (Figure $5 \mathrm{a}$ ). The results show that CD38-specific Nb-CAR-NKs effectively lyse CD38-expressing tumor cells but not the respective CD38ko daughter cell line. Time course analyses revealed that CARDCC happens in a time-dependent manner (Figure 5b). Similarly, the results of titration analyses show that CARDCC occurs in a dose-dependent manner.
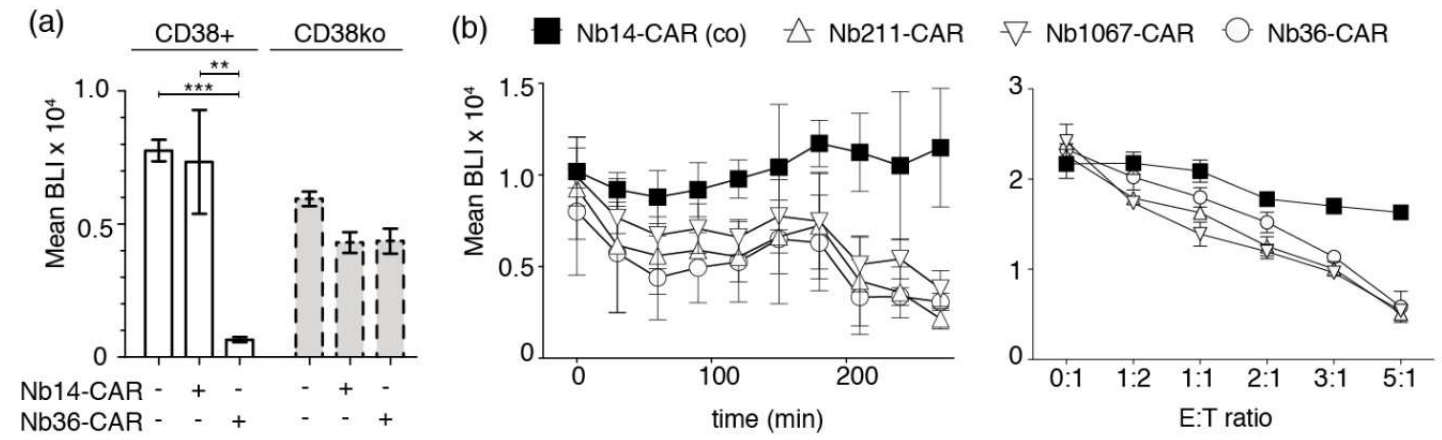

Figure 5. Luminescence-based CARDCC assay of CD38-expressing tumor cells. (a) CD38 ${ }^{+}$or CD38ko CA-46 cells stably transduced with luciferase were incubated for $4 \mathrm{~h}$ at $37^{\circ} \mathrm{C}$ in the absence $(-)$ or presence (+) of NK-92 cells expressing either the control Nb14-CAR or the CD38-specific Nb36-CAR at an effector-to-target ratio of 4:1. Bioluminescence signal intensities (BLI) were measured $20 \mathrm{~min}$ after addition of luciferin. Significance between CD38-specific and control Nb-CAR-NK cells was calculated using unpaired T-test $\left.{ }^{* *}, p<0.01 ;{ }^{* * *}, p<0.005\right)$. (b) The left and right panels show kinetic and dose response analyses of CARDCC toward luciferase-expressing CA-46 cells. Left panel: Nb-CAR-NK cells were co-incubated with CA-46 cells for the indicated times at $37^{\circ} \mathrm{C}$ at an effector-to-target ratio of 1:1 before addition of luciferin and bio-luminometric analysis. Right panel: $\mathrm{Nb}-\mathrm{CAR}-\mathrm{NK}$ cells were co-incubated with CA-46 cells for $4 \mathrm{~h}$ at $37^{\circ} \mathrm{C}$ at the indicated effector-to-target (E:T) ratios before bioluminometry. Results are representative for three similar experiments.

\subsection{A Flow-Cytometric CARDCC}

We next set out to monitor CARDCC by flow cytometry. As a means to distinguish target from effector cells, we labeled $\mathrm{Nb}$-CAR-NK cells with the fluorescent dye eFluor450. To monitor cell death, we used the DNA staining dye propidium iodide, which is excluded by living cells. We co-incubated eFluor450-labeled $\mathrm{Nb}$-CAR-NK cells with CA- 46 cells for $4 \mathrm{~h}$ at $37^{\circ} \mathrm{C}$ before addition of propidium iodide and flow-cytometric analysis (Figure 6a). The results confirm that NK cells expressing the CD38-specific Nb36-CAR, but not those expressing the control Nb14-CAR, indeed effectively lyse CD38-expressing tumor cells. CARDCC caused both-staining by propidium iodide and a decrease in forward light scatter (FSC). The latter likely reflects the shrinkage of lysed cells. 
(a)

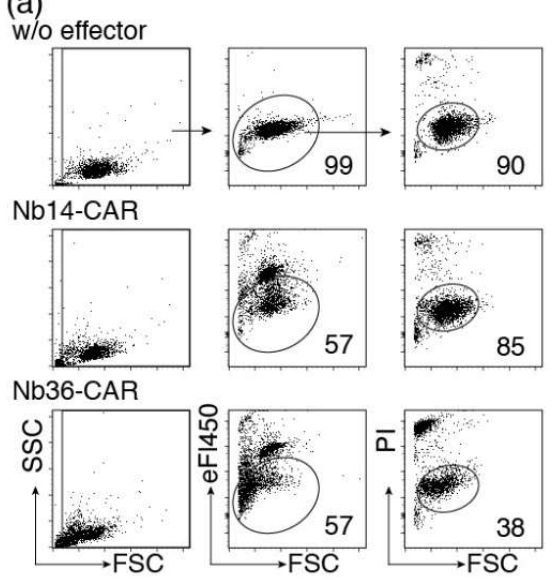

(b)
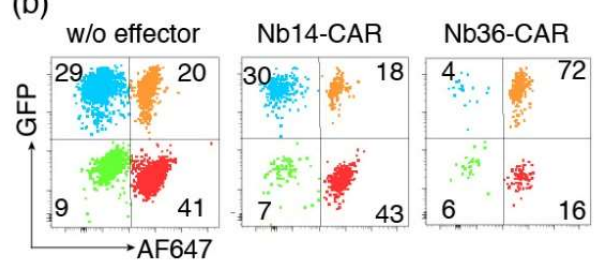

(c)
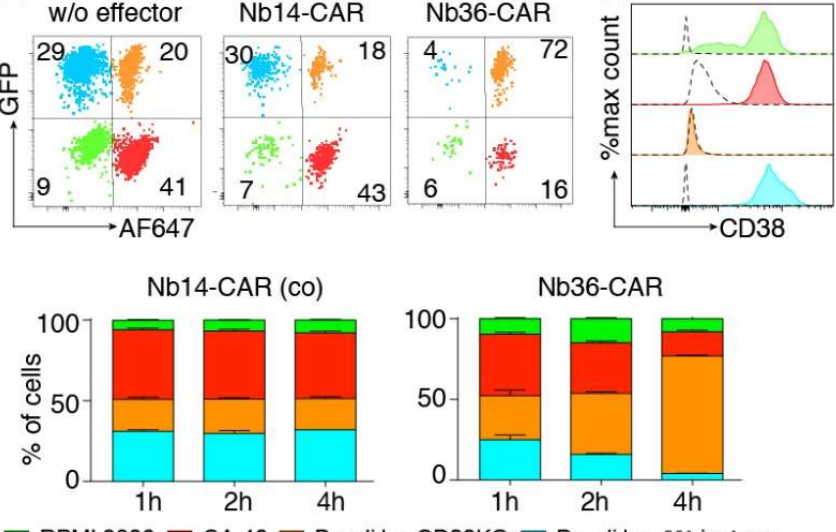

Figure 6. Flow-cytometric CARDCC assay of CD38-expressing tumor cells. (a) CA-46 cells were co-incubated for $4 \mathrm{~h}$ at $37{ }^{\circ} \mathrm{C}$ without (w/o) or with eFluor450-labeled NK-92 cells expressing either the control $\mathrm{Nb} 14-\mathrm{CAR}$ or the CD38-specific Nb36-CAR in an effector to target ratio of 1:1. Cells were analyzed by flow cytometry $10 \mathrm{~min}$ after addition of propidium iodide. Numbers indicate the percentage of cells in the gated population. (b) A mixed population of four color-coded cell lines was incubated for $1-4 \mathrm{~h}$ at $37^{\circ} \mathrm{C}$ with or without (w/o) eFluor450-labeled Nb-CAR-NK cells at an effector-to-target ratio of 1:1. CD38 $8^{\text {lo }}$ RPMI-8226 cells were unlabeled. CD38 ${ }^{\text {hi }}$ CA-46 cells were pre-labeled with Alexa Fluor 647. CD38 ${ }^{+}$and CD38ko Daudi luc cells were GFP positive, CD38ko Daudi cells were pre-labeled with AlexaFluor647. Gating was performed on live (PI-negative), target (eF450-negative) cells. (c) To detect cell surface levels of CD38, the target cells were stained with AlexaFluor647-conjugated MU1067-hcAb and with an AlexaFluor647-conjugated control hcAb (dashed lines). Color coding of cells is as in (b). Results are representative for three similar experiments.

We next set out to determine whether $\mathrm{Nb}-\mathrm{CAR}-\mathrm{NK}$ cells could specifically deplete CD38-expressing cells in mixed populations of CD38 ${ }^{+}$and CD38- cells. To this end we co-incubated CD38 ${ }^{+}$and CD38ko Daudi cells, CD38hi CA-46 cells and CD38 ${ }^{\text {lo }}$ RPMI-8226 cells for 1-4 h with eFluor450-labeled $\mathrm{Nb}-\mathrm{CAR}-\mathrm{NK}$ cells (Figure 6b). The four target cell lines were distinguished on the basis of GFP expression (Daudi luc and Daudi luc CD38ko) and pre-labeling of cells with Alexa Fluor 647 (CA-46 and Daudi luc cells). The results show that CD38-specific but not control CAR-NK cells specifically deplete CD38 expressing cells.

\subsection{CD38-Directed Nb-CAR-NKs Specifically Deplete $C D 38^{+} / C D 56^{+}$Myeloma Cells from Primary Human Bone Marrow Samples}

In the final set of experiments we assessed CARDCC against primary multiple myeloma cells from bone marrow samples of eight human myeloma patients. Myeloma cells were identified by their high levels of cell surface CD38 (and CD56 or CD319). The percentage of such cells in the patient samples ranged from 1.4 to $16.9 \%$ (mean $7.1 \%$ ). We excluded dead cells on the basis of low FSC and staining by PacO. Effector Nb-CAR-NK cells were excluded by staining with eFluor450. In order to permit quantification of absolute cell numbers we added counting beads to the samples (Figure 7a). We found that incubation of bone marrow samples with CD38-specific Nb-CAR-NK cells mediates a significantly higher loss of myeloma cells than incubation with control Nb-CAR-NK cells (Figure 7b). 
(a)

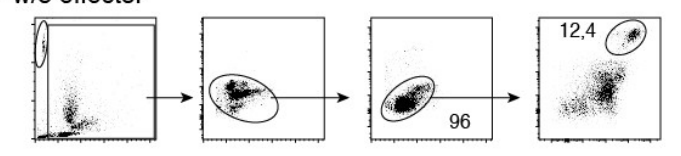

$\mathrm{Nb} 14-\mathrm{CAR}$
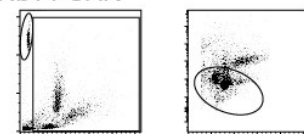

Nb211-CAR
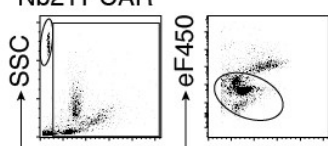
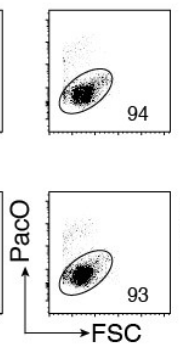
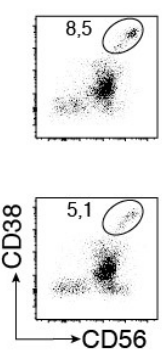

(b)

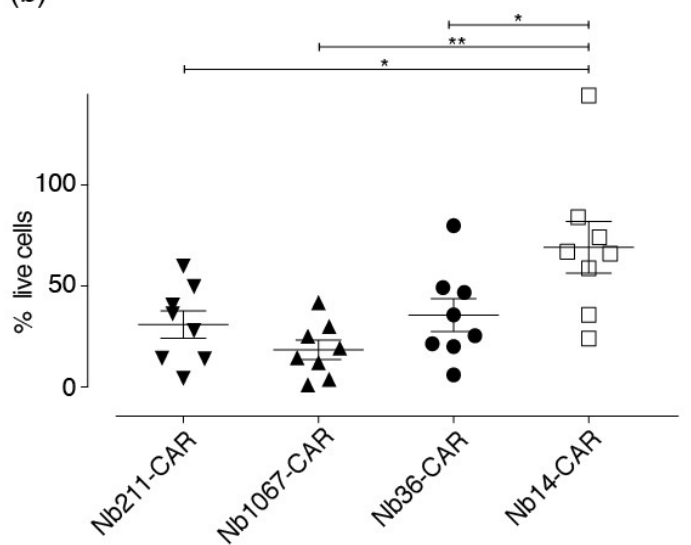

Figure 7. CD38-specific $\mathrm{Nb}-\mathrm{CAR}-\mathrm{NK}$ s deplete $\mathrm{CD} 38^{+}$myeloma cells. Primary human bone marrow cells were co-incubated for $4 \mathrm{~h}$ at $37^{\circ} \mathrm{C}$ without (w/o) or with eFluor450-labeled NK-92 cells expressing either a control CAR (Nb14) or a CD38-specific CAR (Nb211) in an effector to target ratio of 1:1. Cells were counterstained with fluorochrome conjugated $\alpha \mathrm{CD} 56, \alpha \mathrm{CD} 319$, and a CD38-specific Nb that binds a non-overlapping epitope to that of the $\mathrm{Nb}$ contained in the CAR before analysis by flow cytometry. (a) Beads (SSC ${ }^{\text {hi }} / \mathrm{FSC}^{\mathrm{lo}}$ ), effector cells $\left(\mathrm{eF} 450^{+}\right)$, and dead cells $\left(\mathrm{PacO}^{\text {hi }} / \mathrm{FSC}^{\text {lo }}\right.$ ) were excluded by gating. Numbers indicate the percentage of cells in the gated population. Results are representative for eight similar experiments (one for each of eight patient samples). (b) The number of surviving myeloma cells $\left(\mathrm{CD} 38^{+} / \mathrm{CD} 6^{+}\right.$or $\left.\mathrm{CD} 38^{+} / \mathrm{CD} 319^{+}\right)$were determined with the aid of cell counting beads. Percentages of surviving myeloma cells were calculated relative to the number of surviving myeloma cells in the absence of effector cells (set at 100\%). Gating was performed as in (a). Significance between CD38-specific and control $\mathrm{Nb}$-CAR-NK cells was calculated using unpaired T-test ${ }^{*}, p<0.05$; **, $p<0.01)$

\section{Discussion}

Our results demonstrate that CD38-specific nanobodies provide effective ligands for chimeric antigen receptors. We show that the established human NK-92 cell line can be retrovirally transduced to stably express nanobody-based CARs. CAR-expressing NK cells, as a permanent cell line, have the potential to serve as an "off-the-shelf" reagent $[16,23]$. NK-92 cells were originally isolated from the peripheral blood of a 50-year-old patient with non-Hodgkin Lymphoma in 1992 [24]. These cells resemble the phenotype of an activated NK cell. In phase-I trials, NK-92 cells were found to have a high safety profile and to induce only mild graft-versus-host-disease [25,26].

Our results support previous studies demonstrating the potential of nanobody-based CARs [27-30]. Here we used CARs constructed from nanobodies directed against three different epitopes of CD38. All of these mediated effective CARDCC indicate that these epitopes of CD38 are accessible to the CARs. The results further suggest that there is not a preferred epitope of CD38 for CAR-mediated cytotoxicity.

Our data are also in line with those of previous studies using CD38-specific CARs based on scFvs and $\mathrm{Nbs}$ (Figure 8 ). Mihara et al. and Drent et al. fused the single-chain variable fragment of CD38-specific $m A$ bs via the hinge and transmembrane domains of CD8a to the signal transduction domains of 4-1BB and CD3 [ [31,32]. An et al. used a similar architecture for a nanobody-based CAR [27]. More recently, Drent et al. replaced the 4-1BB costimulatory domain with a CD28-costimulatory domain and co-expressed 4-1BBL to provide additional 4-1BB signaling [33]. Our CAR-constructs in turn use the IgG4 hinge and the $\mathrm{CD} 28$ transmembrane region as a bridge to the signal transduction domains of CD28, 4-1BB, and CD3 . In all cases, cysteine residues in the extracellular linker likely mediated the formation of covalently linked dimers. It remains to be determined whether the efficacy of these constructs can be enhanced further by adjusting the length of the extracellular stalk, preventing disulfide formation, e.g., by site directed mutagenesis of cysteine to serine, and/or by replacing the 
signal transduction modules derived from the T-cell surface proteins with signal transduction modules derived from NK cell surface proteins.
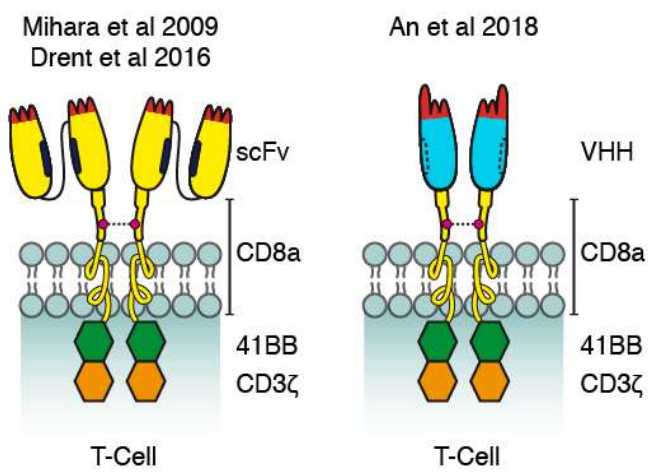

Drent et al 2019

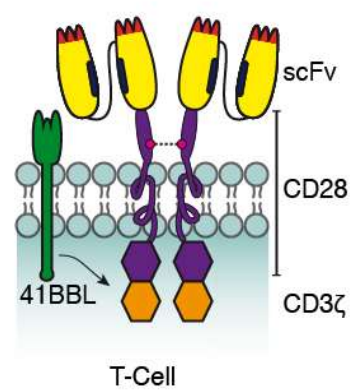

current study

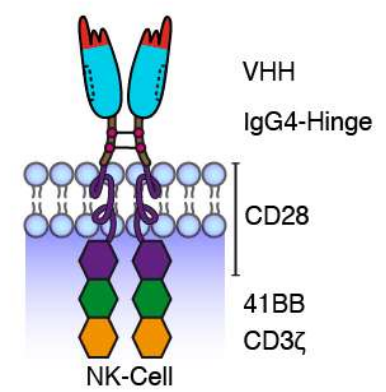

Figure 8. Comparison of CD38-specific CARs used in the current and previous studies. Schematic diagrams illustrating the structures of CD38-specific conventional and $\mathrm{Nb}$-based CARs used in this study to transduce NK-92 cells and in previous studies to transduce T cells. The first conventional and $\mathrm{Nb}$-based CARs reported consisted of a CD38-specific scFv or $\mathrm{Nb}$ connected via the hinge and transmembrane domains of CD8a to the signal transduction domains of 4-1BB and CD3 3 [31,32]. Recently, a more effective conventional CAR was reported carrying the hinge, transmembrane, and signal transduction domains of CD28 and a separate expression-cassette for 4-1BBL transduced into $\mathrm{T}$ cells. The $\mathrm{Nb}-\mathrm{CAR}$ of the current study carries the IgG4 hinge and three cytosolic signal transduction domains.

The interaction between CAR-expressing effector cells and target cells expressing the cognate antigen presumably involves multivalent binding, i.e., the interaction of effector and target cells might encompass the simultaneous engagement of many CARs and cognate antigens on the respective surfaces of effector and target cells. Because non-tumor cells also express CD38, it would be beneficial for cancer therapy if $\mathrm{Nb}$-CAR-NK cells would preferentially deplete CD38 ${ }^{\text {high }}$ cells. We found that RPMI cells with slightly lower levels of CD38 were more resistant to CARDCC after $2 \mathrm{~h}$ of co-incubation with $\mathrm{Nb}$-CAR-NK cells. However, we cannot exclude that other cell surface proteins such as MHCI or inhibitory NK cell receptors influence the susceptibility to CARDCC.

The affinity of the ligand-binding domain of the CAR may also influence the relative cytotoxicity of CAR-expressing cells to target cells that express different levels of the target antigen [34-36]. A low-affinity CAR may preferentially direct CAR-expressing T cells or NK cell to tumor cells that express high surface levels of the target antigen, while sparing normal cells that express lower levels of the target antigen, thereby reducing the potential off target toxicity of CAR-T and CAR-NK cells. Since nanobodies consist of only a single immunoglobulin domain, it will likely be technically easier to modulate the affinity of a nanobody-based CAR than that of a scFv-based CAR [7,8,11].

The specificity may be enhanced by co-targeting of distinct targets, i.e., to two membrane proteins that are co-expressed on tumor cells [37]. This would reduce the unwanted binding of CAR-T or CAR-NK cells to normal cells that express only one of these two target proteins. Based on their excellent solubility and reformating capacity, nanobodies may prove more suitable than conventional scFvs for such approaches [38]. 


\section{Conclusions}

Nanobody-based chimeric antigen receptors ( $\mathrm{Nb}$-CARs) may provide a basis for the clinical development of novel therapeutics to target CD38-expressing tumor cells.

Author Contributions: Conceptualization, B.F., P.B. and F.K.-N.; methodology, J.H., K.R., S.C., K.S., B.A.; validation, K.P., J.L.R., N.B.; investigation, J.H., K.S.; resources, N.K., T.H., G.S.; writing-original draft preparation, J.H., F.K.-N.; writing—review and editing, K.R., F.H., G.A., B.F., P.B.; supervision, B.F., P.B., F.K.-N.; project administration, P.B., F.K.-N.; funding acquisition, B.F., P.B., F.K.-N. All authors have read and agreed to the published version of the manuscript.

Funding: This work was supported by a grant of the Wilhelm Sander-Foundation to P.B. and F.K.-N. (2012.113.2), by a grant of the Deutsche Forschungsgemeinschaft to F.K.-N. (SFB1192, B5), by a grant of the José Carreras Leukämie-Stiftung and the Deutsche Gesellschaft für Hämatologie und Onkologie to K.P., and by a grant of German Cancer Aid (111303) to B.F.

Acknowledgments: The authors thank the FACS Core facility of the UKE for excellent support.

Conflicts of Interest: K.S., P.B., and F.K.-N. are co-inventors on a patent application on CD38-specific nanobodies. F.H. and F.K.-N. receive a share of antibody and protein sales via MediGate $\mathrm{GmbH}$, a wholly owned subsidiary of the University Medical Center Hamburg-Eppendorf. The funders had no role in the design of the study; in the collection, analyses, or interpretation of data; in the writing of the manuscript, or in the decision to publish the results.

\section{References}

1. Deaglio, S.; Aydin, S.; Vaisitti, T.; Bergui, L.; Malavasi, F. CD38 at the junction between prognostic marker and therapeutic target. Trends Mol. Med. 2008, 14, 210-218. [CrossRef] [PubMed]

2. Horenstein, A.L.; Bracci, C.; Morandi, F.; Malavasi, F. CD38 in adenosinergic pathways and metabolic re-programming in human multiple myeloma cells: In-tandem insights from basic science to therapy. Front. Immunol. 2019, 10, 760. [CrossRef] [PubMed]

3. Morandi, F.; Horenstein, A.L.; Costa, F.; Giuliani, N.; Pistoia, V.; Malavasi, F. CD38: A target for immunotherapeutic approaches in multiple myeloma. Front. Immunol. 2018, 9, 2722. [CrossRef] [PubMed]

4. $\quad$ Lonial, S.; Weiss, B.M.; Usmani, S.Z.; Singhal, S.; Chari, A.; Bahlis, N.J.; Belch, A.; Krishnan, A.; Vescio, R.A.; Mateos, M.V.; et al. Daratumumab monotherapy in patients with treatment-refractory multiple myeloma (SIRIUS): An open-label, randomised, phase 2 trial. Lancet 2016, 387, 1551-1560. [CrossRef]

5. van de Donk, N.; Richardson, P.G.; Malavasi, F. CD38 antibodies in multiple myeloma: Back to the future. Blood 2018, 131, 13-29. [CrossRef] [PubMed]

6. Wong, S.W.; Comenzo, R.L. CD38 Monoclonal Antibody Therapies for Multiple Myeloma. Clin. Lymphoma Myeloma Leuk. 2015, 15, 635-645. [CrossRef]

7. Muyldermans, S. Nanobodies: Natural single-domain antibodies. Annu. Rev. Biochem. 2013, 82, $775-797$. [CrossRef]

8. Wesolowski, J.; Alzogaray, V.; Reyelt, J.; Unger, M.; Juarez, K.; Urrutia, M.; Cauerhff, A.; Danquah, W.; Rissiek, B.; Scheuplein, F.; et al. Single domain antibodies: Promising experimental and therapeutic tools in infection and immunity. Med. Microbiol. Immunol. 2009, 198, 157-174. [CrossRef]

9. Conrath, K.E.; Wernery, U.; Muyldermans, S.; Nguyen, V.K. Emergence and evolution of functional heavy-chain antibodies in Camelidae. Dev. Comp. Immunol. 2003, 27, 87-103. [CrossRef]

10. Bannas, P.; Hambach, J.; Koch-Nolte, F. Nanobodies and Nanobody-Based Human Heavy Chain Antibodies As Antitumor Therapeutics. Front. Immunol. 2017, 8, 1603. [CrossRef]

11. Ingram, J.R.; Schmidt, F.I.; Ploegh, H.L. Exploiting Nanobodies' Singular Traits. Annu. Rev. Immunol. 2018, 36, 695-715. [CrossRef] [PubMed]

12. Bannas, P.; Koch-Nolte, F. Perspectives for the Development of CD38-Specific Heavy Chain Antibodies as Therapeutics for Multiple Myeloma. Front. Immunol. 2018, 9, 2559. [CrossRef] [PubMed]

13. Fumey, W.; Koenigsdorf, J.; Kunick, V.; Menzel, S.; Schutze, K.; Unger, M.; Schriewer, L.; Haag, F.; Adam, G.; Oberle, A.; et al. Nanobodies effectively modulate the enzymatic activity of CD38 and allow specific imaging of CD38(+) tumors in mouse models in vivo. Sci. Rep. 2017, 7, 14289. [CrossRef] [PubMed] 
14. Schriewer, L.; Schutze, K.; Petry, K.; Hambach, J.; Fumey, W.; Koenigsdorf, J.; Baum, N.; Menzel, S.; Rissiek, B.; Riecken, K.; et al. Nanobody-based CD38-specific heavy chain antibodies induce killing of multiple myeloma and other hematological malignancies. Theranostics 2019. (In press) [CrossRef]

15. Schutze, K.; Petry, K.; Hambach, J.; Schuster, N.; Fumey, W.; Schriewer, L.; Rockendorf, J.; Menzel, S.; Albrecht, B.; Haag, F.; et al. CD38-Specific Biparatopic Heavy Chain Antibodies Display Potent Complement-Dependent Cytotoxicity Against Multiple Myeloma Cells. Front. Immunol. 2018, 9, 2553. [CrossRef]

16. Klingemann, H.; Boissel, L.; Toneguzzo, F. Natural Killer Cells for Immunotherapy - Advantages of the NK-92 Cell Line over Blood NK Cells. Front. Immunol. 2016, 7, 91. [CrossRef]

17. Weber, K.; Bartsch, U.; Stocking, C.; Fehse, B. A multicolor panel of novel lentiviral "gene ontology" (LeGO) vectors for functional gene analysis. Mol. Ther. 2008, 16, 698-706. [CrossRef]

18. Weber, K.; Mock, U.; Petrowitz, B.; Bartsch, U.; Fehse, B. Lentiviral gene ontology (LeGO) vectors equipped with novel drug-selectable fluorescent proteins: New building blocks for cell marking and multi-gene analysis. Gene Ther. 2010, 17, 511-520. [CrossRef]

19. Koch-Nolte, F.; Reyelt, J.; Schossow, B.; Schwarz, N.; Scheuplein, F.; Rothenburg, S.; Haag, F.; Alzogaray, V.; Cauerhff, A.; Goldbaum, F.A. Single domain antibodies from llama effectively and specifically block T cell ecto-ADP-ribosyltransferase ART2.2 in vivo. FASEB J. 2007, 21, 3490-3498. [CrossRef]

20. Schambach, A.; Mueller, D.; Galla, M.; Verstegen, M.M.; Wagemaker, G.; Loew, R.; Baum, C.; Bohne, J. Overcoming promoter competition in packaging cells improves production of self-inactivating retroviral vectors. Gene Ther. 2006, 13, 1524-1533. [CrossRef]

21. Schambach, A.; Bohne, J.; Chandra, S.; Will, E.; Margison, G.P.; Williams, D.A.; Baum, C. Equal potency of gammaretroviral and lentiviral SIN vectors for expression of O6-methylguanine-DNA methyltransferase in hematopoietic cells. Mol. Ther. 2006, 13, 391-400. [CrossRef]

22. Frigyesi, I.; Adolfsson, J.; Ali, M.; Christophersen, M.K.; Johnsson, E.; Turesson, I.; Gullberg, U.; Hansson, M.; Nilsson, B. Robust isolation of malignant plasma cells in multiple myeloma. Blood 2014, 123, 1336-1340. [CrossRef] [PubMed]

23. Hodgins, J.J.; Khan, S.T.; Park, M.M.; Auer, R.C.; Ardolino, M. Killers 2.0: NK cell therapies at the forefront of cancer control. J. Clin. Invest. 2019, 129, 3499-3510. [CrossRef] [PubMed]

24. Gong, J.H.; Maki, G.; Klingemann, H.G. Characterization of a human cell line (NK-92) with phenotypical and functional characteristics of activated natural killer cells. Leukemia 1994, 8, 652-658. [PubMed]

25. Arai, S.; Meagher, R.; Swearingen, M.; Myint, H.; Rich, E.; Martinson, J.; Klingemann, H. Infusion of the allogeneic cell line NK-92 in patients with advanced renal cell cancer or melanoma: A phase I trial. Cytotherapy 2008, 10, 625-632. [CrossRef] [PubMed]

26. Tonn, T.; Schwabe, D.; Klingemann, H.G.; Becker, S.; Esser, R.; Koehl, U.; Suttorp, M.; Seifried, E.; Ottmann, O.G.; Bug, G. Treatment of patients with advanced cancer with the natural killer cell line NK-92. Cytotherapy 2013, 15, 1563-1570. [CrossRef]

27. An, N.; Hou, Y.N.; Zhang, Q.X.; Li, T.; Zhang, Q.L.; Fang, C.; Chen, H.; Lee, H.C.; Zhao, Y.J.; Du, X. Anti-Multiple Myeloma Activity of Nanobody-Based Anti-CD38 Chimeric Antigen Receptor T Cells. Mol. Pharm. 2018, 15, 4577-4588. [CrossRef]

28. Bakhtiari, S.H.; Rahbarizadeh, F.; Hasannia, S.; Ahmadvand, D.; Iri-Sofla, F.J.; Rasaee, M.J. Anti-MUC1 nanobody can redirect T-body cytotoxic effector function. Hybrid 2009, 28, 85-92. [CrossRef]

29. Hassani, M.; Hajari Taheri, F.; Sharifzadeh, Z.; Arashkia, A.; Hadjati, J.; van Weerden, W.M.; Modarressi, M.H.; Abolhassani, M. Construction of a chimeric antigen receptor bearing a nanobody against prostate a specific membrane antigen in prostate cancer. J. Cell Biochem. 2019, 120, 10787-10795. [CrossRef]

30. Xie, Y.J.; Dougan, M.; Jailkhani, N.; Ingram, J.; Fang, T.; Kummer, L.; Momin, N.; Pishesha, N.; Rickelt, S.; Hynes, R.O.; et al. Nanobody-based CAR T cells that target the tumor microenvironment inhibit the growth of solid tumors in immunocompetent mice. Proc. Natl. Acad. Sci. USA 2019, 116, 7624-7631. [CrossRef]

31. Drent, E.; Groen, R.W.; Noort, W.A.; Themeli, M.; Lammerts van Bueren, J.J.; Parren, P.W.; Kuball, J.; Sebestyen, Z.; Yuan, H.; de Bruijn, J.; et al. Pre-clinical evaluation of CD38 chimeric antigen receptor engineered $\mathrm{T}$ cells for the treatment of multiple myeloma. Haematologica 2016, 101, 616-625. [CrossRef] [PubMed] 
32. Mihara, K.; Yanagihara, K.; Takigahira, M.; Imai, C.; Kitanaka, A.; Takihara, Y.; Kimura, A. Activated T-cell-mediated immunotherapy with a chimeric receptor against CD38 in B-cell non-Hodgkin lymphoma. J. Immunother. 2009, 32, 737-743. [CrossRef] [PubMed]

33. Drent, E.; Poels, R.; Ruiter, R.; van de Donk, N.; Zweegman, S.; Yuan, H.; de Bruijn, J.; Sadelain, M.; Lokhorst, H.M.; Groen, R.W.J.; et al. Combined CD28 and 4-1BB Costimulation Potentiates Affinity-tuned Chimeric Antigen Receptor-engineered T Cells. Clin. Cancer Res. 2019, 25, 4014-4025. [CrossRef] [PubMed]

34. Chmielewski, M.; Hombach, A.; Heuser, C.; Adams, G.P.; Abken, H. T cell activation by antibody-like immunoreceptors: Increase in affinity of the single-chain fragment domain above threshold does not increase T cell activation against antigen-positive target cells but decreases selectivity. J. Immunol. 2004, 173, 7647-7653. [CrossRef]

35. Drent, E.; Themeli, M.; Poels, R.; de Jong-Korlaar, R.; Yuan, H.; de Bruijn, J.; Martens, A.C.M.; Zweegman, S.; van de Donk, N.; Groen, R.W.J.; et al. A Rational Strategy for Reducing On-Target Off-Tumor Effects of CD38-Chimeric Antigen Receptors by Affinity Optimization. Mol. Ther. 2017, 25, 1946-1958. [CrossRef]

36. Hudecek, M.; Lupo-Stanghellini, M.T.; Kosasih, P.L.; Sommermeyer, D.; Jensen, M.C.; Rader, C.; Riddell, S.R. Receptor affinity and extracellular domain modifications affect tumor recognition by ROR1-specific chimeric antigen receptor T cells. Clin. Cancer Res. 2013, 19, 3153-3164. [CrossRef]

37. Perna, F.; Berman, S.H.; Soni, R.K.; Mansilla-Soto, J.; Eyquem, J.; Hamieh, M.; Hendrickson, R.C.; Brennan, C.W.; Sadelain, M. Integrating Proteomics and Transcriptomics for Systematic Combinatorial Chimeric Antigen Receptor Therapy of AML. Cancer Cell 2017, 32, 506-519. [CrossRef]

38. De Munter, S.; Ingels, J.; Goetgeluk, G.; Bonte, S.; Pille, M.; Weening, K.; Kerre, T.; Abken, H.; Vandekerckhove, B. Nanobody Based Dual Specific CARs. Int. J. Mol. Sci. 2018, 19. [CrossRef]

(C) 2020 by the authors. Licensee MDPI, Basel, Switzerland. This article is an open access article distributed under the terms and conditions of the Creative Commons Attribution (CC BY) license (http://creativecommons.org/licenses/by/4.0/). 